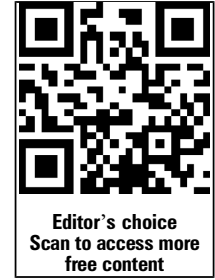

- Additional material is published online only. To view please visit the journal online (http://dx.doi.org/10.1136/ heartjnl-2014-306663).

For numbered affiliations see end of article.

\section{Correspondence to}

Dr Yoosoo Chang, Department of Occupational and Environmental Medicine, Kangbuk Samsung Hospital, 108 Pyung dong, Jongro-Gu, Seoul, 110-746, Republic of Korea; yoosoo.chang@gmail. com

Received 15 August 2014 Revised 8 January 2015 Accepted 15 January 2015 Published Online First 2 March 2015

\title{
Coffee consumption and coronary artery calcium in young and middle-aged asymptomatic adults
}

\author{
Yuni Choi, ${ }^{1}$ Yoosoo Chang, ${ }^{1,2,3}$ Seungho Ryu, 1,2,3 Juhee Cho, ${ }^{1,4,5}$ Sanjay Rampal, 5,6 \\ Yiyi Zhang, ${ }^{5}$ Jiin Ahn, ${ }^{1}$ Joao A C Lima, ${ }^{7}$ Hocheol Shin, ${ }^{1,8}$ Eliseo Guallar ${ }^{5}$
}

\begin{abstract}
Objective To investigate the association between regular coffee consumption and the prevalence of coronary artery calcium (CAC) in a large sample of young and middle-aged asymptomatic men and women. Methods This cross-sectional study included 25138 men and women (mean age 41.3 years) without clinically evident cardiovascular disease who underwent a health screening examination that included a validated food frequency questionnaire and a multidetector $\mathrm{CT}$ to determine CAC scores. We used robust Tobit regression analyses to estimate the CAC score ratios associated with different levels of coffee consumption compared with no coffee consumption and adjusted for potential confounders.
\end{abstract}

Results The prevalence of detectable CAC (CAC score $>0$ ) was $13.4 \%$ ( $n=3364)$, including $11.3 \%$ prevalence for CAC scores $1-100(n=2832)$, and $2.1 \%$ prevalence for CAC scores $>100(n=532)$. The mean \pm SD consumption of coffee was $1.8 \pm 1.5$ cups/day. The multivariate-adjusted CAC score ratios ( $95 \% \mathrm{Cls}$ ) comparing coffee drinkers of $<1,1-<3,3-<5$, and $\geq 5$ cups/day to non-coffee drinkers were 0.77 (0.49 to 1.19 ), 0.66 (0.43 to 1.02), 0.59 (0.38 to 0.93$)$, and 0.81 (0.46 to 1.43 ), respectively ( $p$ for quadratic trend $=0.02$ ). The association was similar in subgroups defined by age, sex, smoking status, alcohol consumption, status of obesity, diabetes, hypertension, and hypercholesterolaemia.

Conclusions In this large sample of men and women apparently free of clinically evident cardiovascular disease, moderate coffee consumption was associated with a lower prevalence of subclinical coronary atherosclerosis.

\section{INTRODUCTION}

The effect of coffee consumption on cardiovascular health has remained controversial. ${ }^{1}$ In spite of earlier concerns about a potential increase in cardiovascular disease (CVD) risk associated with coffee consumption, a recent meta-analysis of 36 prospective studies showed that moderate coffee consumption was associated with a decreased risk of CVD, with the strongest reduction at 3-5 cups/ day. ${ }^{2}$ Coffee consumption has been associated with improved insulin sensitivity, ${ }^{3-5}$ reduced low density lipoprotein cholesterol (LDL-C) oxidation, ${ }^{6}{ }^{7}$ and reduced risk of type 2 diabetes. ${ }^{8} 9$ However, coffee consumption has also been associated with increased cholesterol concentrations ${ }^{10}{ }^{11}$ and acute pressor effects, although the relationship of coffee consumption to the risk of hypertension is still unclear. $^{1}$
Coronary artery calcium (CAC) detected by cardiac CT is a subclinical marker of coronary atherosclerosis that predicts future coronary heart disease $(\mathrm{CHD})^{12}$ in a wide range of ages, including asymptomatic young adults. ${ }^{13}$ CAC provides information on underlying pathologic changes in coronary arteries and thus allows for a detailed assessment of the early stages of CVD, which is distinct from the occurrence of plaque rupture and thrombosis in clinical events. ${ }^{14}$ Only two studies have examined the relationship between coffee consumption and CAC, with inconsistent results. ${ }^{15}{ }^{16}$ We therefore examined the association between coffee consumption and the presence of CAC in a large sample of asymptomatic men and women attending a health screening examination.

\section{METHODS \\ Study population}

The Kangbuk Samsung Health Study is a cohort study of Korean men and women who underwent comprehensive annual or biennial examinations at the Kangbuk Samsung Hospital Total Healthcare Centers in Seoul or Suwon, South Korea. ${ }^{17}$ The population for this cross-sectional analysis included all participants $(n=30485)$ who completed a food frequency questionnaire (FFQ) and who underwent a comprehensive health screening examination including cardiac CT for CAC scoring between March 2011 and April 2013. Annual or biennial health screening examinations are widely performed in Korea, as health examinations are mandatory for all workers under the Industrial Safety and Health Law. CAC scoring has become a common CVD screening test in Korea. ${ }^{17}$ After participants had completed selfadministered questionnaires, all examinations including CT scan for CAC scoring were performed.

We excluded 437 participants with a history of CVD, 474 participants who had implausible estimated energy consumption levels (beyond 3 SDs from the $\log _{\mathrm{e}}$-transformed mean energy consumption), 1824 participants with missing information on coffee consumption, and 3371 participants with missing information on smoking status. The final sample size for the analysis was 25138 participants (figure 1).

This study was approved by the Institutional Review Board of the Kangbuk Samsung Hospital. The requirement of informed consent was waived because we used non-identified retrospective data routinely collected during the health screening process. 


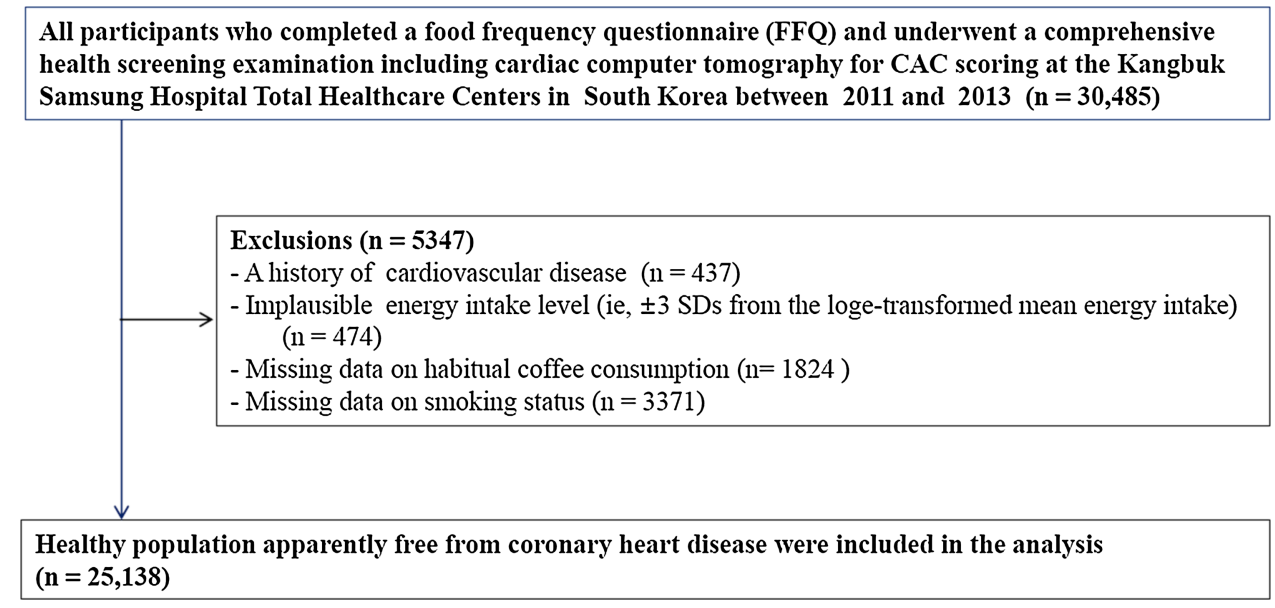

Figure 1 Flow diagram of the included study participants. CAC, coronary artery calcium.

\section{Assessment of coffee consumption}

Usual dietary consumption was assessed using a 103-item selfadministered FFQ designed for use in Korea. ${ }^{18}$ The reproducibility and validity of the nutrient information provided by this FFQ have been evaluated among 124 Korean subjects aged 40-70 years. Participants were asked how often, on average, they consumed each type of food or beverage during the past year. The FFQ had three predefined categories of portion size, ranging from small to large, and nine predefined categories of frequency, ranging from never or seldom to $\geq$ three times per day for foods, and from never or seldom to $\geq$ five times per day for beverages. Participants were also asked to report the consumption period (ie, 3, 6, 9, or 12 months) for seasonal consumption of fruits. The FFQ asked about total coffee consumption without differentiating between caffeinated and decaffeinated coffee. However, decaffeinated coffee is not yet widely popular in Korea. ${ }^{19}$ A recipe, portion size, and nutrient database was constructed with a food composition table from the Korean Nutrition Society. ${ }^{20}$

\section{Measurement of CAC}

All CT scans were obtained with a Lightspeed VCT XTe 64 slice multidetector CT (MDCT) scanner (GE Healthcare, Tokyo, Japan) with a standard scanning protocol using $2.5 \mathrm{~mm}$ thickness, $400 \mathrm{~ms}$ rotation time, $120 \mathrm{kV}$ tube voltage, and a $124 \mathrm{mAS}(310 \mathrm{~mA} \times 0.4 \mathrm{~s})$ tube current under ECG-gated dose modulation. Quantitative CAC scores were calculated using the method proposed by Agatston et al. ${ }^{21}$ The interobserver and intraobserver reliability values of CAC scores were both excellent, with intraclass correlation coefficients of 0.99 . If more than one CAC measurement was available for a participant during the study period, the initial measurement was used.

\section{Assessment of other variables}

Data on medical history, medication use, family history, physical activity, alcohol consumption, smoking habits, and sociodemographic characteristics were collected through a selfadministered questionnaire, whereas the anthropometric parameters and blood pressure were measured by trained staff during the health examinations. ${ }^{17}$ Physical activity levels were assessed by the Korean-validated version of the short form of the International Physical Activity Questionnaire (IPAQ); participants were classified as inactive, minimally active, and health enhancing physically active (HEPA). ${ }^{22}$ Hypertension was defined as systolic blood pressure $(\mathrm{SBP}) \geq 140 \mathrm{~mm} \mathrm{Hg}$, diastolic blood pressure $\geq 90 \mathrm{~mm} \mathrm{Hg}$, a participant report of a previous diagnosis, or current antihypertensive medication use.

Blood specimens were sampled from the antecubital vein after at least a $10 \mathrm{~h}$ fast. Measurements of serum biochemical parameters, including serum concentrations of glucose, haemoglobin A1c (HbA1c), total cholesterol, triglycerides, LDL-C, high density lipoprotein cholesterol (HDL-C), and serum high sensitivity $\mathrm{C}$ reactive protein (hsCRP) have been described in detail elsewhere. ${ }^{17}$ Diabetes mellitus was defined as fasting serum glucose $\geq 126 \mathrm{mg} / \mathrm{dL}, \mathrm{HbA} 1 \mathrm{c} \geq 6.5 \%$, self-report of a previous diagnosis, or use of blood glucose lowering agents. Hypercholesterolaemia was defined as a serum total cholesterol $\geq 240 \mathrm{mg} / \mathrm{dL}$, self-report of a previous diagnosis, or current use of cholesterol lowering medication. The Laboratory Medicine Department at Kangbuk Samsung Hospital has been accredited by the Korean Society of Laboratory Medicine and participates annually in inspections and surveys by the Korean Association of Quality Assurance for Clinical Laboratories and the College of American Pathologists Proficiency Testing programme.

\section{Statistical analysis}

Habitual coffee consumption was categorised as none, $<1$, $1-<3,3-<5$, and $\geq 5$ cups/day. For the primary analyses, we modelled $\log _{\mathrm{e}}(\mathrm{CAC}$ score +1$)$ using robust Tobit regression model with the Huber-White estimation of SEs. ${ }^{14}$ We $\log _{\mathrm{e}}$ -transformed the CAC scores since their distribution was skewed to the right and used Tobit models because the CAC scores were left-censored at 0 Agatston units (non-detectable CAC scores). Tobit models were used to estimate CAC score ratios and their 95\% CIs for each category of coffee consumption. CAC score ratios represent the ratio of the geometric means of CAC scores comparing each category of coffee consumption to the group with no coffee consumption. In a secondary analyses, we categorised CAC scores as a dichotomous variable (CAC score $>0$ compared with CAC score 0 ) and used logistic regression to estimate the ORs for detectable CAC according to the categories of coffee consumption.

Statistical models were initially adjusted for age, sex, centre, and year of screening examination (model 1) and then further adjusted for potential confounding factors determined a priori, ${ }^{23}$ including education level (high school graduate or less, community college or university graduate, graduate school or higher, and unknown), physical activity level (inactive, 
minimally active, HEPA, and unknown), smoking status (never, former, and current), BMI (body mass index, $\mathrm{kg} / \mathrm{m}^{2}$ ), parental history of CHD (yes vs no), alcohol consumption (none, $<5,5-$ $<20, \geq 20 \mathrm{~g} /$ day, and unknown), total energy consumption (kcal/ day), and consumption of fruits, vegetables, and red and processed meats (quartiles, g/day) (model 2). Further adjusting for tea consumption (including black, green tea, and any other teas) did not materially change the results. We additionally adjusted for potential intermediate variables, including SBP, fasting serum glucose, total cholesterol, triglycerides, and HDL-C (model 3). We conducted quadratic trend tests by including linear and quadratic terms of the median coffee consumption within each coffee consumption category as continuous variables in the regression models, and statistical significance was evaluated using Wald tests.

We conducted subgroup analyses by age $(<40$ vs $\geq 40$ years), sex (men vs women), smoking (non-current vs current smoker), BMI ( $<25$ vs $\geq 25 \mathrm{~kg} / \mathrm{m}^{2}$ ), alcohol drinking (non-drinker vs drinker), diabetes (yes vs no), hypertension (yes vs no), and hypercholesterolaemia (yes vs no). We tested for statistical interactions by using Wald tests for the product term of coffee consumption categories with the stratification variable.

In sensitivity analyses, we repeated the multivariate analyses after excluding those who did not consume coffee because of the possibility that they could represent individuals with unfavourable health conditions, and each category was compared with the reference category of coffee drinkers of $<1$ cup/day. All analyses were conducted using STATA V.12.1 (Stata Corp, College Station, Texas, USA). Statistical tests were a two-sided test, and $\mathrm{p}<0.05$ was considered statistically significant.

\section{RESULTS}

The average \pm SD age of study participants was $41.3 \pm 7.5$ years, and $83.7 \%$ of participants were male. The prevalence of CAC scores $>0$ was $13.4 \%(n=3364)$, including $11.3 \%$ prevalence for CAC scores $1-100(n=2832)$, and $2.1 \%$ prevalence for CAC scores $>100(n=532)$. The mean \pm SD consumption of coffee was $1.8 \pm 1.5$ cups/day. Compared with non-coffee drinkers, individuals in the highest categories of coffee consumption were more likely to be older, men, and current smokers and to have higher education, less frequent vigorous-intensity physical activity, obesity, and hypercholesterolaemia, and to have a lower proportion of hypertension (table 1). Compared to non-coffee drinkers, those with high coffee consumption also had higher concentrations of fasting serum glucose, HbA1c, total cholesterol, triglycerides, and LDL-C, and lower HDL-C and SBP, and they had a higher consumption of total energy, red and processed meats, alcohol, and a lower consumption of fruits and vegetables.

Table 1 Characteristics of study participants by categories of coffee consumption

\begin{tabular}{|c|c|c|c|c|c|}
\hline \multirow[b]{2}{*}{ Characteristics } & \multicolumn{5}{|c|}{ Daily coffee consumption } \\
\hline & $\begin{array}{l}\text { None } \\
(n=1381)\end{array}$ & $\begin{array}{l}<1 \text { cup } \\
(n=6666)\end{array}$ & $\begin{array}{l}1-<3 \text { cups } \\
(n=9849)\end{array}$ & $\begin{array}{l}3-<5 \text { cups } \\
(n=6084)\end{array}$ & $\begin{array}{l}\geq 5 \text { cups } \\
(n=1158)\end{array}$ \\
\hline Age (years) & $41.1(8.8)$ & $40.6(8.0)$ & $41.4(7.4)$ & $42(6.7)$ & $42.1(6.7)$ \\
\hline Males (\%) & 73.8 & 82.2 & 82.6 & 88.5 & 87.9 \\
\hline College graduation or higher $(\%)$ & 77.7 & 81.3 & 83.3 & 83.3 & 83.2 \\
\hline Current smoker (\%) & 13.8 & 23.6 & 28.7 & 40 & 48.9 \\
\hline HEPA (\%) & 9.6 & 7.4 & 8 & 7.1 & 7.2 \\
\hline Obesity (\%) & 31.9 & 37.2 & 38.2 & 43.2 & 46.9 \\
\hline Diabetes (\%) & 6.4 & 6.2 & 5.8 & 5.8 & 7.2 \\
\hline Hypertension (\%) & 19 & 16.4 & 16.6 & 16.6 & 15.5 \\
\hline Hypercholesterolaemia (\%) & 21.8 & 24.6 & 25.8 & 27.7 & 29.1 \\
\hline Parental history of CHD (\%) & 7.9 & 7.1 & 7.5 & 8.1 & 7.3 \\
\hline SBP $(\mathrm{mm} \mathrm{Hg})$ & $115.0(13.5)$ & $115.6(13.2)$ & $114.9(13.2)$ & $114.9(12.6)$ & $114.6(12.4)$ \\
\hline $\mathrm{DBP}(\mathrm{mm} \mathrm{Hg})$ & $73.7(10.3)$ & $74.0(10.4)$ & $73.7(10.5)$ & $73.9(10.1)$ & $73.5(9.9)$ \\
\hline Glucose (mmol/L) & $5.4(0.81)$ & $5.5(0.9)$ & $5.5(0.9)$ & $5.5(0.9)$ & $5.5(0.9)$ \\
\hline $\mathrm{HbA1c}(\%)$ & $5.67(0.45)$ & $5.69(0.53)$ & $5.69(0.52)$ & $5.71(0.55)$ & $5.72(0.53)$ \\
\hline Total cholesterol (mmol/L) & $5.1(0.9)$ & $5.2(0.9)$ & $5.2(0.9)$ & $5.3(0.9)$ & $5.4(0.9)$ \\
\hline Triglycerides (mmol/L) & $1.2(0.8-1.7)$ & $1.3(0.9-1.9)$ & $1.3(0.9-1.9)$ & $1.3(0.9-1.9)$ & $1.4(1.0-2.0)$ \\
\hline $\mathrm{HDL}-\mathrm{C}(\mathrm{mmol} / \mathrm{L})$ & $1.4(0.4)$ & $1.4(0.3)$ & $1.4(0.3)$ & $1.4(0.3)$ & $1.3(0.3)$ \\
\hline LDL-C (mmol/L) & $3.2(0.8)$ & $3.3(0.8)$ & $3.3(0.8)$ & $3.4(0.8)$ & $3.5(0.8)$ \\
\hline hsCRP (mg/L) & $0.5(0.3-0.9)$ & $0.5(0.3-1.0)$ & $0.5(0.3-1.0)$ & $0.5(0.3-1.1)$ & $0.5(0.3-1.1)$ \\
\hline \multicolumn{6}{|l|}{ Daily dietary consumption } \\
\hline Total energy (Kcal/day) & $1570.8(728.4)$ & $1519.7(653.7)$ & $1604.4(636.7)$ & $1720.4(676.1)$ & $1840.9(722.6)$ \\
\hline Vegetables (g/day) & $96.6(112.9)$ & $80.2(76.7)$ & $86.0(84.1)$ & $85.4(78.5)$ & $91.1(94.6)$ \\
\hline Fruits (g/day) & $220.0(343.1)$ & $185.0(213.4)$ & $195.6(205.8)$ & $197.0(207.1)$ & $210.6(250.1)$ \\
\hline Red and processed meat (g/day) & $55.6(52.9)$ & $60.8(57.1)$ & $61.1(54.5)$ & $67.5(59.7)$ & $74.7(72.4)$ \\
\hline Alcohol (g/day) & $3.9(0.6-16.9)$ & $6.0(1.8-19.2)$ & $7.0(2.3-20.8)$ & $7.9(2.7-22.6)$ & $8.6(2.8-24.3)$ \\
\hline Tea drinker, $0>$ cup/day $(\%)^{*}$ & 54.0 & 72.1 & 67.2 & 65.3 & 59.4 \\
\hline
\end{tabular}


Table 2 Coronary artery calcium (CAC) score ratios* (95\% Cls) by categories of coffee consumption

\begin{tabular}{|c|c|c|c|c|c|c|}
\hline & \multicolumn{5}{|c|}{ Daily coffee consumption } & \multirow[b]{2}{*}{ P for quadratic trendt } \\
\hline & $\begin{array}{l}\text { None } \\
(n=1381)\end{array}$ & $\begin{array}{l}<1 \text { cup } \\
(n=6666)\end{array}$ & $\begin{array}{l}1-<3 \text { cups } \\
(\mathrm{n}=9849)\end{array}$ & $\begin{array}{l}3-<5 \text { cups } \\
(n=6084)\end{array}$ & $\begin{array}{l}\geq 5 \text { cups } \\
(n=1158)\end{array}$ & \\
\hline Model $1 \ddagger$ & 1.00 & 0.86 (0.55 to 1.34$)$ & 0.82 (0.53 to 1.26$)$ & $0.78(0.50$ to 1.22$)$ & 1.17 (0.66 to 2.06 ) & 0.20 \\
\hline Model $2 \S$ & 1.00 & 0.77 (0.49 to 1.19 ) & 0.66 (0.43 to 1.02$)$ & 0.59 (0.38 to 0.93$)$ & 0.81 (0.46 to 1.43 ) & 0.02 \\
\hline Model 39 & 1.00 & 0.76 (0.49 to 1.18 ) & $0.68(0.44$ to 1.05$)$ & 0.62 (0.39 to 0.97$)$ & 0.79 (0.45 to 1.40$)$ & 0.07 \\
\hline
\end{tabular}

${ }^{*}$ Derived from robust Tobit regression models using $\log _{\mathrm{e}}(\mathrm{CAC}+1)$ as the outcome.

tDerived from the quadratic trend tests by including a linear and quadratic term of the median coffee consumption value within each group in the regression models.

¥Model 1: Adjusted for age, sex, centre, and year of screening examination.

§Model 2: Model 1 with additional adjustments for education level (high school graduate or less, community college or university graduate, graduate school or higher, and unknown), physical activity (inactive, minimally active, health enhancing physical activity, and unknown), smoking (never, former, and current smoker), body mass index (kg/m²), parental history of coronary heart disease (yes vs no), alcohol consumption (none, $<5,5-<20, \geq 20 \mathrm{~g} /$ day, and unknown), total energy consumption (kcal/d), consumption of fruits, vegetables, and red and processed meats (quartiles, g/day).

१Model 3: Model 2 with additional adjustments for potential intermediate variables, including systolic blood pressure, fasting serum glucose, total cholesterol, triglyceride, and high density lipoprotein cholesterol.

In a model that adjusted for age, sex, centre, and year of screening examination (table 2; model 1), the CAC ratios $(95 \%$ CI) comparing coffee drinkers of $<1,1-<3,3-<5$, and $\geq 5$ cups/day to non-coffee drinkers were 0.86 (0.55 to 1.34$), 0.82$ (0.53 to 1.26$), 0.78$ (0.50 to 1.22$)$, and 1.77 (0.66 to 2.06$)$, respectively ( $\mathrm{p}$ for quadratic trend $=0.20$ ). Further adjusting for potential confounders strengthened the inverse association between coffee consumption and CAC, most likely due to smoking status. In multivariable adjusted models (model 2), the corresponding estimates were 0.77 (0.49 to 1.19$), 0.66$ (0.43 to $1.02), 0.59$ (0.38 to 0.93$)$, and 0.81 (0.46 to 1.43 ), respectively ( $\mathrm{p}$ for quadratic trend $=0.02$ ). The findings were similar after adjustment for SBP, fasting serum glucose, total cholesterol, triglycerides, and HDL-C (model 3).

The results from logistic regression models using categories of CAC scores were consistent with the Tobit models. The multivariable adjusted ORs (95\% CIs) for CAC score $>0$ comparing coffee drinkers of $<1,1-<3,3-<5$, and $\geq 5$ cups/day to noncoffee drinkers were 0.90 (0.74 to 1.10$), 0.85$ (0.71 to 1.03$)$, 0.80 ( 0.66 to 0.97 ), and 0.94 ( 0.73 to 1.20$)$, respectively ( $\mathrm{p}$ for quadratic trend $=0.02$ ) (table 3 , model 2), with little change after adjustment for potential intermediate variables. The significant quadratic trend between coffee consumption and detectable CAC scores was also evident after adjusting for potential confounders (figure 2).
The association was consistent in subgroup analyses, and there were no statistically significant interactions (data not shown; $\mathrm{p}$ for interaction $>0.05$ ). In sensitivity analyses where we excluded non-coffee drinkers, the multivariable adjusted CAC score ratios $(95 \%$ CIs) comparing coffee drinkers of $1-<3$, $3-<5$, and $\geq 5$ cups/day to coffee drinkers of $<1$ cup/day were 0.86 (0.68 to 1.09$), 0.77$ (0.59 to 1.00$)$, and 1.04 (0.67 to 1.62 ), respectively (model 2 ).

\section{DISCUSSION}

In this large sample of Korean men and women apparently free of clinically evident CVD, coffee consumption was associated with a lower prevalence of CAC, a marker of subclinical coronary atherosclerosis. The association was U-shaped, with participants drinking $3-<5$ cups/day having the lowest prevalence of subclinical coronary atherosclerosis. The association was similar after adjustment for cardiovascular risk factors or potential mediators. Our findings are consistent with a recent body of literature showing that moderate coffee consumption may be inversely associated with cardiovascular events.

The relationship between coffee consumption and CVD has been extensively investigated with conflicting results. However, several large prospective studies and reviews have concluded that habitual coffee consumption is associated with decreased risk of cardiovascular events, and this

Table $3 \mathrm{OR}^{*}(95 \% \mathrm{Cls})$ for the presence of coronary artery calcium (CAC) score by categories of coffee consumption

\begin{tabular}{|c|c|c|c|c|c|c|}
\hline & \multicolumn{5}{|c|}{ Daily coffee consumption } & \multirow[b]{2}{*}{ P for quadratic trendt } \\
\hline & $\begin{array}{l}\text { None } \\
(n=1381)\end{array}$ & $\begin{array}{l}<1 \text { cup } \\
(n=6666)\end{array}$ & $\begin{array}{l}1-<3 \text { cups } \\
(\mathrm{n}=9849)\end{array}$ & $\begin{array}{l}3-<5 \text { cups } \\
(n=6084)\end{array}$ & $\begin{array}{l}\geq 5 \text { cups } \\
(n=1158)\end{array}$ & \\
\hline \multicolumn{7}{|c|}{ CAC score $>0$ vs 0} \\
\hline No. of cases & 181 & 844 & 1300 & 853 & 186 & \\
\hline Model $1 \ddagger$ & 1.00 & 0.95 (0.78 to 1.15$)$ & 0.93 (0.77 to 1.12 ) & $0.90(0.74$ to 1.09$)$ & 1.10 (0.86 to 1.40$)$ & 0.15 \\
\hline Model $2 \S$ & 1.00 & $0.90(0.74$ to 1.10$)$ & 0.85 (0.71 to 1.03 ) & 0.80 (0.66 to 0.97$)$ & 0.94 (0.73 to 1.20$)$ & 0.02 \\
\hline Model 3ף & 1.00 & $0.89(0.73$ to 1.09$)$ & 0.86 (0.71 to 1.04$)$ & 0.81 (0.66 to 0.98$)$ & 0.93 (0.72 to 1.19$)$ & 0.06 \\
\hline \multicolumn{7}{|c|}{$\begin{array}{l}\text { *Derived from logistic regression models using dichotomous CAC score as outcome variable (CAC score }>0 \text { compared to CAC score } 0 \text { ). } \\
\text { †Derived from the quadratic trend tests by including a linear and quadratic term of the median coffee consumption value within each group in the regression models. } \\
\text { ¥Model 1: Adjusted for age, sex, centre, and year of screening examination. } \\
\text { \$Model 2: Model } 1 \text { with additional adjustments for education level (high school graduate or less, community college or university graduate, graduate school or higher, and unknown), } \\
\text { physical activity (inactive, minimally active, health enhancing physical activity, and unknown), smoking (never, former, and current smoker), body mass index (kg/m²), parental history of } \\
\text { coronary heart disease (yes vs no), alcohol consumption (none, }<5,5-<20, \geq 20 \mathrm{~g} / \text { day, and unknown), total energy consumption (kcal/day), consumption of fruits, vegetables, and red } \\
\text { and processed meats (quartiles, g/day). } \\
\text { १Model 3: Model } 2 \text { with additional adjustments for potential intermediate variables, including systolic blood pressure, fasting serum glucose, total cholesterol, triglyceride, and high } \\
\text { density lipoprotein cholesterol. }\end{array}$} \\
\hline
\end{tabular}




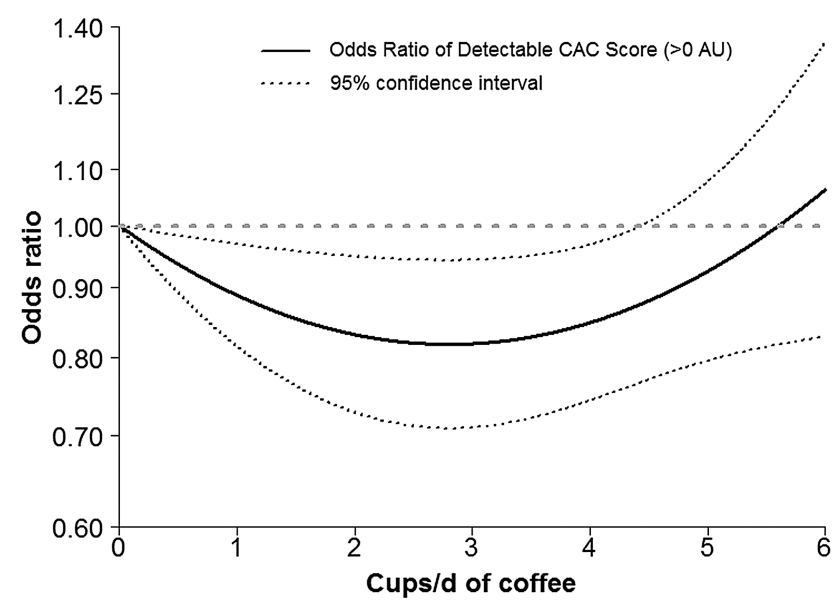

Figure 2 OR of detectable coronary artery calcium scores (CAC score $>0$ ) by coffee consumption. The curves represent the adjusted OR of detectable CAC scores ( $>0$ Agatston unit) and coffee consumption. The dose response association of coffee consumption was estimated by using a linear and a quadratic term for coffee consumption in the multivariable logistic regression. The model adjusted for age, sex, centre, and year of screening examination, education level (high school graduate or less, community college or university graduate, graduate school or higher, and unknown), physical activity level (inactive, minimally active, health enhancing physically active, and unknown), smoking status (never, former, and current), body mass index $\left(\mathrm{kg} / \mathrm{m}^{2}\right)$, parental history of coronary heart disease (yes vs no), alcohol consumption (none, $<5,5-<20, \geq 20 \mathrm{~g} /$ day, and unknown), total energy consumption (kcal/day), and consumption of fruits, vegetables, and red and processed meats (quartiles, g/day). A similar trend was observed in robust Tobit regression models of CAC ratios.

association was most evident for moderate coffee consumption levels. ${ }^{9} 24-26$ A recent meta-analysis of 36 prospective studies also showed that moderate coffee consumption was associated with decreased CVD risk, with the largest reduction at 3-5 cups/day. ${ }^{2}$

Long term coffee consumption was associated with improved endothelial function. ${ }^{27}$ Only two studies, however, have investigated the association between coffee consumption and CAC. ${ }^{15}{ }^{16}$ In the Rotterdam study $(\mathrm{n}=1570),{ }^{15}$ a moderate (>3-4 cups/day) to high coffee ( $>4$ cups/day) consumption was associated with a decreased prevalence of a CAC score $>400$ compared with $\leq 3$ drinks/day in women, but an increased risk was observed in non-smoking men. In the Coronary Artery Risk Development in Young Adults (CARDIA) study $(\mathrm{n}=5115),{ }^{16} \mathrm{caf}-$ feinated and decaffeinated coffee consumption was not associated with coronary calcification or with progression over 5 years of follow-up. In the Rotterdam and CARDIA studies, ${ }^{15}{ }^{16}$ measurements of coffee intake were taken several years before CAC measurements (an average of 7 years in the Rotterdam Study and at least 8 years in CARDIA), which may have induced misclassification of exposure status. In our study, coffee consumption was assessed on the same day of the screening exam or in the previous 14 days, minimising misclassification of exposure and avoiding biased reporting as dietary information was collected before CAC measurements. The inconsistent findings across studies could also be attributable to other methodological issues, including differences in sample size, control for potential confounding factors, measures of coffee consumption, study population (age, ethnicity, and sex composition), or prevalence of CAC.
Although none of the potential mediating variables fully explained the relationship between coffee and the presence of CAC, several mechanisms have been suggested for the association between moderate coffee and cardiovascular risk. Chronic coffee consumption has been associated with reduced risk of type 2 diabetes, ${ }^{8}{ }^{9}$ a strong risk factor for atherosclerosis. In addition, coffee drinking might improve insulin sensitivity and $\beta$-cell function, presumably due to the presence of antioxidant compounds in the coffee. ${ }^{3-5}$ Although acute coffee consumption might increase blood pressure, this effect seems to disappear with chronic coffee consumption and large prospective studies do not support the hypothesis that high coffee consumption increases the risk of hypertension. ${ }^{1}$ In our data, we observed an inverse relationship between regular coffee consumption and SBP, but adjusting for SBP and other CVD risk factors did not materially change the association between coffee consumption and the presence of CAC. Coffee also contains phenolic compounds with high antioxidant activity that might prevent LDL-C oxidation, ${ }^{6} 7$ and coffee consumption was inversely associated with markers of inflammation ${ }^{28}$ and endothelial dysfunction. $^{29}$ On the other hand, coffee, particularly unfiltered coffee, has shown a cholesterol raising effect. ${ }^{10}{ }^{11}$ Further research is needed to understand the potential effects of moderate coffee consumption on CVD risk, which are likely to be complex and at least partly independent of traditional CVD risk factors.

The present study has several limitations. First, the crosssectional design limits the possibility of establishing causal inferences as we cannot rule out reverse causation or residual confounding. However, we examined the association in asymptomatic individuals free of reported CVD and used as outcome CAC, a marker of subclinical disease not recognised by study participants. Furthermore, the estimates did not change after adjusting for a variety of CVD risk factors or after excluding non-coffee drinkers who might abstain from coffee drinking due to unfavourable health status. Second, exposure assessment of coffee and other lifestyle factors was based on self-report and subject to measurement error, which would tend to attenuate the observed associations. Third, we were unable to differentiate between caffeinated and decaffeinated coffee. Decaffeinated coffee, however, is not popular among Koreans. ${ }^{19}$ In addition, a previous study did not show substantial differences in the association between caffeinated and decaffeinated coffee and CAC. ${ }^{16}$ Finally, our results were based on a sample of relatively healthy, young and middle-aged, educated Koreans, and might not be generalisable to other populations.

Our study also has several strengths. The large sample size provides sufficient power to detect the association between coffee drinking and CAC scores while controlling for a wide range of potential confounders. Data collection in the Kangbuk Samsung Health Study is subject to careful standardisation and quality control. Also, we had information on a large number of CVD risk factors that could be used to adjust for potential confounders in multivariable models.

In conclusion, we found that moderate daily coffee consumption was associated with decreased prevalence of CAC in a large sample of asymptomatic adults free of CVD. Our study adds to a growing body of evidence suggesting that coffee consumption might be inversely associated with CVD risk. Further research is warranted to confirm our findings and establish the biological basis of coffee's potential preventive effects on coronary artery disease. 


\section{Key messages}

What is already known on this subject?

The relationship between coffee consumption and cardiovascular disease is controversial. Several large prospective studies and reviews have concluded that habitual coffee consumption may be associated with decreased risk of cardiovascular events, and this association was most evident for moderate coffee consumption levels.

\section{What might this study add?}

In this large sample of Korean men and women free of clinically evident cardiovascular disease, coffee consumption was associated with a lower prevalence of coronary artery calcium, a marker of subclinical coronary atherosclerosis. The association was U-shaped, with participants drinking $3-<5$ cups/day having the lowest prevalence of subclinical coronary atherosclerosis.

\section{How might this impact on clinical practice?}

Moderate daily coffee consumption was inversely associated with subclinical coronary atherosclerosis. Large scale and randomised controlled studies are needed to prove causation.

\author{
Author affiliations \\ ${ }^{1}$ Center for Cohort Studies, Total Healthcare Screening Center, Kangbuk Samsung \\ Hospital, Sungkyunkwan University, School of Medicine, Seoul, Republic of Korea \\ ${ }^{2}$ Department of Occupational and Environmental Medicine, Kangbuk Samsung \\ Hospital, Sungkyunkwan University, School of Medicine, Seoul, Republic of Korea \\ ${ }^{3}$ Department of Clinical Research Design and Evaluation, SAIHST, Sungkyunkwan \\ University, Seoul, Republic of Korea \\ ${ }^{4}$ Department of Health Sciences and Technology, SAIHST, Sungkyunkwan University, \\ Seoul, Republic of Korea \\ ${ }^{5}$ Departments of Epidemiology and Medicine, and Welch Center for Prevention, \\ Epidemiology, and Clinical Research, Johns Hopkins Bloomberg School of Public \\ Health, Baltimore, MD, USA \\ ${ }^{6}$ Department of Social and Preventive Medicine, Julius Centre University of Malaya, \\ Faculty of Medicine, University of Malaya. Kuala Lumpur, Malaysia \\ ${ }^{7}$ Division of Cardiology, Johns Hopkins University School of Medicine, Baltimore, \\ MD, USA \\ ${ }^{8}$ Department of Family Medicine, Kangbuk Samsung Hospital, Sungkyunkwan \\ University School of Medicine, Seoul, Republic of Korea
}

Contributors All authors were involved in the preparation of the manuscript, have read the manuscript, agree with the analyses of the data and the conclusions reached in the manuscript, and are accountable for all aspects of the work.

\section{Competing interests None.}

Patient consent Requirement for informed consent was waived because the study used non-identified retrospective data routinely collected during health screening process.

Ethics approval Ethics approval was provided by the Institutional Review Board of the Kangbuk Samsung Hospital.

Provenance and peer review Not commissioned; externally peer reviewed.

\section{REFERENCES}

1 Riksen NP, Rongen GA, Smits P. Acute and long-term cardiovascular effects of coffee: implications for coronary heart disease. Pharmacol Ther 2009;121:185-91.

2 Ding M, Bhupathiraju SN, Satija A, et al. Long-term coffee consumption and risk of cardiovascular disease: a systematic review and a dose-response meta-analysis of prospective cohort studies. Circulation 2014;129:643-59.

3 Loopstra-Masters RC, Liese AD, Haffner SM, et al. Associations between the intake of caffeinated and decaffeinated coffee and measures of insulin sensitivity and beta cell function. Diabetologia 2011;54:320-8.
4 Arnlov J, Vessby B, Riserus U. Coffee consumption and insulin sensitivity. JAMA 2004;291:1199-201.

5 Bruce CR, Carey AL, Hawley JA, et al. Intramuscular heat shock protein 72 and heme oxygenase- 1 mRNA are reduced in patients with type 2 diabetes: evidence that insulin resistance is associated with a disturbed antioxidant defense mechanism. Diabetes 2003;52:2338-45.

6 Gomez-Ruiz JA, Leake DS, Ames JM. In vitro antioxidant activity of coffee compounds and their metabolites. J Agric Food Chem 2007;55:6962-9.

7 Natella F, Nardini M, Belelli F, et al. Coffee drinking induces incorporation of phenolic acids into $L D L$ and increases the resistance of $L D L$ to ex vivo oxidation in humans. Am J Clin Nutr 2007;86:604-9.

8 Huxley R, Lee CM, Barzi F, et al. Coffee, decaffeinated coffee, and tea consumption in relation to incident type 2 diabetes mellitus: a systematic review with meta-analysis. Arch Intern Med 2009;169:2053-63.

9 Freedman ND, Park Y, Abnet CC, et al. Association of coffee drinking with total and cause-specific mortality. N Eng/ J Med 2012;366:1891-904.

10 Ranheim T, Halvorsen B. Coffee consumption and human health--beneficial or detrimental?--mechanisms for effects of coffee consumption on different risk factors for cardiovascular disease and type 2 diabetes mellitus. Mol Nutr Food Res 2005;49:274-84

11 Jee $\mathrm{SH}$, He J, Appel $\mathrm{L}$, et al. Coffee consumption and serum lipids: a meta-analysis of randomized controlled clinical trials. Am J Epidemiol 2001;153:353-62.

12 Budoff MJ, Achenbach S, Blumenthal RS, et al. Assessment of coronary artery disease by cardiac computed tomography: a scientific statement from the American Heart Association Committee on Cardiovascular Imaging and Intervention, Council on Cardiovascular Radiology and Intervention, and Committee on Cardiac Imaging, Council on Clinical Cardiology. Circulation 2006;114:1761-91.

13 LaMonte MJ, FitzGerald SJ, Church TS, et al. Coronary artery calcium score and coronary heart disease events in a large cohort of asymptomatic men and women. Am J Epidemiol 2005;162:421-9.

14 Reilly MP, Wolfe ML, Localio AR, et al. Coronary artery calcification and cardiovascular risk factors: impact of the analytic approach. Atherosclerosis 2004;173:69-78

15 van Woudenbergh GJ, Vliegenthart R, van Rooij FJ, et al. Coffee consumption and coronary calcification: the Rotterdam Coronary Calcification Study. Arterioscler Thromb Vasc Biol 2008;28:1018-23.

16 Reis JP, Loria CM, Steffen LM, et al. Coffee, decaffeinated coffee, caffeine, and tea consumption in young adulthood and atherosclerosis later in life: the CARDIA study. Arterioscler Thromb Vasc Biol 2010;30:2059-66.

17 Chang Y, Kim BK, Yun KE, et al. Metabolically-healthy obesity and coronary artery calcification. J Am Coll Cardiol 2014;63:2679-86.

18 Ahn Y, Kwon E, Shim JE, et al. Validation and reproducibility of food frequency questionnaire for Korean genome epidemiologic study. Eur J Clin Nutr 2007;61:1435-41.

19 The U.S. Agricultural Trade Office, U.S. Embassy Seoul, Korea, Coffee market brief update Korea-Republic of. http://gain.fas.usda.gov/Recent\%20GAIN\% 20Publications/Coffee\%20Market\%20Brief\%20Update_Seoul\%20ATO_Korea\% 20-\%20Republic\%20of_2-5-2013.pdf (5 May 2014)

20 The Korean Nutrition Society. Food value: nutrient composition table for foods. Seoul: The Korean Nutrition Society, 2006.

21 Agatston AS, Janowitz WR, Hildner FJ, et al. Quantification of coronary artery calcium using ultrafast computed tomography. J Am Coll Cardiol 1990;15:827-32.

22 Oh JY, Kim BS, Kang JH. Validity and reliability of Korean Version of International Physical Activity Questionnaire (IPAQ) Short Form. J Korean Acad Fam Med 2007;28:532-41.

23 Cornelis MC, El-Sohemy A. Coffee, caffeine, and coronary heart disease. Curr Opin Clin Nutr Metab Care 2007;10:745-51.

24 Mostofsky E, Rice MS, Levitan EB, et al. Habitual coffee consumption and risk of heart failure: a dose-response meta-analysis. Circ Heart Fail 2012;5:401-5.

25 Larsson SC, Orsini N. Coffee consumption and risk of stroke: a dose-response meta-analysis of prospective studies. Am J Epidemiol 2011;174:993-1001.

26 O'Keefe JH, Bhatti SK, Patil HR, et al. Effects of habitual coffee consumption on cardiometabolic disease, cardiovascular health, and all-cause mortality. J Am Coll Cardiol 2013;62:1043-51.

27 Siasos G, Oikonomou E, Chrysohoou C, et al. Consumption of a boiled Greek type of coffee is associated with improved endothelial function: the Ikaria study. Vascu Med 2013;18:55-62.

28 Kempf K, Herder C, Erlund I, et al. Effects of coffee consumption on subclinical inflammation and other risk factors for type 2 diabetes: a clinical trial. Am J Clin Nutr 2010;91:950-7.

29 Lopez-Garcia E, van Dam RM, Qi L, et al. Coffee consumption and markers of inflammation and endothelial dysfunction in healthy and diabetic women. Am J Clin Nutr 2006;84:888-93. 